\title{
Introduction to the Special Issue Psychological Assistance for Public during the Pandemic of COVID-19
}

\author{
Mingyi Qian ${ }^{1}$, Rodney Goodyear ${ }^{2}$, Guangrong Jiang ${ }^{3, *}$ and Zhihong Ren ${ }^{3}$ \\ ${ }^{1}$ School of Psychological and Cognitive Sciences, Beijing Key Laboratory of Behavior and Mental Health, Peking University, \\ Beijing, 100871, China \\ ${ }^{2}$ Department of Counseling and Human Services, University of Redlands, Redlands, CA 92373, USA \\ ${ }^{3}$ School of Psychology, Central China Normal University, Key Laboratory of Adolescent Cyberpsychology and Behavior, Ministry \\ of Education, Key Laboratory of Human Development and Mental Health of Hubei Province, Wuhan, 430079, China \\ *Corresponding Author: Guangrong Jiang. Email: grjiang@yeah.net \\ Received: 24 July 2020; Accepted: 04 August 2020
}

The first reports of a mysterious new coronavirus-related pneumonia began to appear just as people were welcoming in the new year. Within weeks, parts of China were locked-down in response to this highly contagious disease, and by mid-March COVID-19 had spread so much globally that the World Health Organization declared a pandemic. Almost everyone was affected. Travel was restricted, even within cities. Schools, restaurants, sporting events and movie theatres were closed as social gatherings were discouraged or even prohibited. Some people were fortunate enough to be able to work from home using video conferencing technologies; some were forced to continue to work even when that put them at risk for COVID-19 exposure; and some were now unemployed. Some people became seriously ill, some lost loved ones, and a great many have lived with the constant fear of being infected and concerns for their family and jobs.

These conditions have created stress and trauma as people addressed their feelings of threat and helplessness. Mental health professionals, though, began to recognize this almost immediately and began responding by:

1. Conducting research to understand the extent to which the pandemic was changing people's lives, and in what ways;

2. Disseminating psychological knowledge through mass media to help people cope with the effects of the pandemic; and,

3. Developing hotlines and other psychological helping systems.

Three of our guest editing team had first-hand knowledge of these efforts. In fact, Guangrong Jiang and Zhihong Ren were in Wuhan during the outbreak. They met with Mingyi Qian who was in Beijing via Zoom in April, after the first wave of the pandemic was under control in China, to discuss how to share with peers across the world examples of how Chinese mental health professionals had responded to the pandemic. The hope was that lessons learned from those initiatives would be useful to peers in other parts of the world who were experiencing similar challenges. They then were joined by Rod Goodyear from the United States guest edit this a special issue of the International Journal of Mental

This work is licensed under a Creative Commons Attribution 4.0 International License, which permits unrestricted use, distribution, and reproduction in any medium, provided the original work is properly cited. 
Health Promotion (IJMHP). We appreciate this platform that IJMHP provided for sharing the experience and the lessons that the authors in this issue are able to offer.

Two of the nine articles in this issue are from Europe, and the rest from China. Four articles address how the pandemic affected the mental health status and needs of different populations of people, including those on the front-lines as care givers. Cunill et al. [1] report their investigation of the pandemic's effects on the mental health of the medical professionals in Spain who were ones exposed to the COVID-19 patients; Zhu et al. [2] addressed the professional-help needs of the police in Hubei Province, who were performing one of the most essential jobs during the pandemic; Xia et al. [3] reported results of a survey of Chinese international students in a number of western countries on their emotional experience and stress coping during the pandemic; and Chen et al. [4] addressed ways in which an event of this magnitude and type can affect personal world-views, including even the meaning of life.

Five of the articles report responses to the pandemic that mental health professionals developed and implemented. Jia et al. [5] described the experience of building a helpline in China, offering lessons from that experience and suggestions about ways in which the service quality can be maintained on a more ongoing bases; the articles by Bric et al. [6] and Zhao et al. [7] both provide examples of to take advantage of the current resource to build a mental health service during the pandemic; Lin et al. [8] report their findings concerning the quality of mental health services offered by hotlines during the pandemic; and, Zhong et al. [9] reviewed the adaptability of the mental health system in China during the pandemic and reflecting on how to make the system work more efficient.

As they have had to address the challenges that the pandemic has created for them, people have changed in their attitude towards themselves, their relationships and even their lives. The professionals working to help them have themselves been changed as well. Unaddressed trauma can certainly have negative effects, those are well recognized. But Tedeschi et al. [10] have made the case for Post-traumatic Growth (PTG), which can occur as a result of having dealt with a trauma. That is much more likely when the trauma is understood and responded to effectively. It is to be hoped that articles like those in this issue will decrease the negative effects of trauma and perhaps even facilitate PTG.

It is important to recognize, of course, the contexts that affect the work mental health professionals do and the outcomes they obtain. The articles in this special issue focus on the work and experience of authors form different culture in different countries, each of which is affected by culture, politics, economy and social formations. Therefore, although our intention is for these articles to provide information and lessons that are generalizable, we also recognize that readers will need to extrapolate those to their own particular contexts.

Finally, it is important to acknowledge that the COVID-19 pandemic is still affecting the world. As it persists, people are likely to be presented with new issues and challenges. We hope, therefore, that mental health professionals will continue their efforts to better understand and address the emerging needs. We look forward to reading about those efforts in future issues of this and other journals.

\section{References}

1. Cunill, M., Aymerich, M., Serdà, B. C., Patiño-Masó, J. (2020). The impact of COVID-19 on Spanish health professionals: a description of physical and psychological effects. International Journal of Mental Health Promotion, 22(3), 185-198.

2. Zhu, X., Xia, M., Hu, Y., Zhang, L., Lu, Y. (2020). Mental status and psychological needs of Chinese police officers in a highly impacted city during the COVID-19 pandemic. International Journal of Mental Health Promotion, 22(3), 149-157.

3. Xia, M., Duan, C. (2020). Stress coping of Chinese International students in face of COVID 19 pandemic: cultural characteristics. International Journal of Mental Health Promotion, 22(3), 159-172. 
4. Chen, C., Zhang, Y., Xu, A., Chen, X., Lin, J. (2020). Reconstruction of meaning in life: meaning made during the pandemic of COVID-19. International Journal of Mental Health Promotion, 22(3), 173-184.

5. Jia, X., You, L., An, Q. (2020). Specifics and operational procedures of the psychological assistance hotline during the COVID-19 pandemic in China. International Journal of Mental Health Promotion, 22(3), 109-113.

6. Bric, B., Raile, P. (2020). Installing an innovative helpline at the psychotherapeutic outpatient clinic of the Sigmund-Freud-Privat University in Vienna during the COVID-19-crisis. International Journal of Mental Health Promotion, 22(3), 133-147.

7. Zhao, C., Shi, C., Zhang, L., Zhai, Z., Ren, Z. et al. (2020). Establishment of online platform for psychological assistance during a public health emergency. International Journal of Mental Health Promotion, 22(3), 123-132.

8. Lin, X., Swift, J., Cheng, Y., An, Q., Liang, H. et al. (2020). The psychological hotline services quality survey during the pandemic of COVID-19 in mainland China. International Journal of Mental Health Promotion, 22(3), 115-121.

9. Zhong, J., Fan, F., Liu, Y. (2020). Cogitation on the mental health service system during the COVID-19 outbreak in China. International Journal of Mental Health Promotion, 22(3), 199-202.

10. Tedeschi, R. G., Calhoun, L. G. (1995). Trauma and transformation. Newbury Park, CA: SAGE. 